\title{
Injection molding and mechanical properties evaluation of short jute fiber polypropylene reinforced composites
}

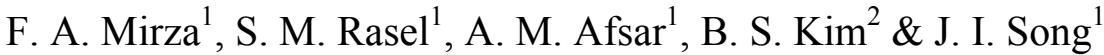 \\ ${ }^{I}$ Department of Mechanical Engineering, Changwon National University, \\ South Korea \\ ${ }^{2}$ Composite Materials Group, KIMS, South Korea
}

\begin{abstract}
Composites with polypropylene (PP) and $2 \mathrm{wt} \% \mathrm{NaOH}$ treated jute fibers were prepared by the injection molding technique. In order to improve the affinity and adhesion between the reinforced jute fiber and the polymer matrix (polypropylene) during manufacturing, maleic anhydride grafted polypropylene (MAPP) as a coupling agent has been employed. Tensile tests were carried out to evaluate the composite mechanical properties. The tensile test results showed the composite to have higher strength and modulus than pure polypropylene (PP). In addition, strength and modulus were found to be influenced by the variation of MAPP contents $(1 \%, 2 \%$, and $3 \%)$. The tensile strengths were improved by $19.3 \%, 21.7 \%$, and $23.8 \%$, respectively, compared with the raw PP. The tensile moduli were improved by $110.7 \%, 122.7 \%$, and $148 \%$, respectively, compared with the raw PP.
\end{abstract}

Keywords: natural fibers, polypropylene, surface treatments, interfacial bonding, mechanical properties.

\section{Introduction}

Over the past decade there has been a growing interest in the use of lignocellulosic fibers as reinforcing elements in polymeric matrices [1-5]. The specific properties of this natural product, namely low cost, lightweight, renewable character, high specific strength and modulus, availability in a variety of forms throughout the world, reactive surface and the possibility to generate 
energy, without residue, after burning at the end of its life-cycle, motivate its association with organic polymers to elaborate composite materials. However, it is well known that different surface properties between the fiber and the matrix, i.e. the former is highly polar and hydrophilic while the latter is, generally, nonpolar and relatively hydrophobic, impose the surface modification of the fibers surface, in order to improve the fiber/polymer compatibility and their interfacial adhesion [6]. Without such a treatment, natural fibers embedded in a polymeric matrix generate unstable interfaces; the stress applied to the fiber/polymer composite is not efficiently transferred from the matrix to the fiber and the beneficial reinforcement effect of the fiber remains underexploited. Likewise, the poor ability of the polymer to wet the fiber hinders the homogeneous dispersion of short fibers within the polymeric matrix [7].

Several strategies of surface modifications aiming at improving the compatibility between cellulose fibers and polymer matrices were recently reviewed [8]. The chemical modification using coupling agents bearing two reactive groups, one of which is likely to react with the $\mathrm{OH}$ function at the fiber surface, whereas the other one is left to copolymerize with the matrix, constitutes a highly interesting way of allowing the establishment of covalent bonding between fibers and matrix, thus leading to materials with high mechanical properties. Many coupling agents have been investigated, namely anhydrides, maleated polymer [9-11], isocyanates [12-14], triazines [15] and alkoxysilanes [16-20], as recently reviewed [8]. Among these different reagents, maleated polypropylene (MaPP) or polyethylene (MaPE) gave significant enhancement in tensile and flexural strength, ranging from $40 \%$ up to $80 \%$, when they are blended with cellulose fibers before mixing with the matrix $[9,11]$.

The surface modification of cellulose fibers with a polypropylene-maleic anhydride copolymer resulted in improved mechanical properties of the cellulose-polypropylene composites as shown by Felix and Gatenholm [21]. Scanning electron microscopy (SEM) studies revealed improved dispersion and adhesion when the fibers were surface modified. Similar studies were reported by Karmaker and Youngquist [22] for jute fiber reinforced polypropylene composites, and by Chen et al. [23] for bamboo fiber reinforced polypropylene composites. Both of these studies used maleic anhydride-grafted polypropylene as the coupling agent.

Jute is one of the most common natural fibers that obtain high tensile modulus and low elongation at break. If the low density $\left(1.3 \mathrm{~g} / \mathrm{cm}^{3}\right)$ of this fiber is taken into consideration, then its specific stiffness and strength are comparable to those of glass fiber. However, according to the references cited in this paper, there are very few reports that pay attention to short jute fiber reinforced composites. One of the important investigations of this study was to manufacture short jute fiber reinforced composites by the injection molding technique, as followed by the evaluation of their mechanical properties. The contribution of a fiber to strengthening the composite performance is considerably high, when the fiber is sufficiently longer than the critical length. On the other hand, the higher the adhesion between fiber and matrix polymer, the shorter is the critical fiber length. This study deals with the short jute fibers $(\sim 2 \mathrm{~mm})$ with respect to the 
ideal situation when the adhesion between the fibers and the matrix polymer can be high. Generally, as hydrophilic jute fibers do not adhere well to PP, which is hydrophobic in nature, Maleic anhydride grafted polypropylene (MAPP) was employed to improve the bonding between the fibers and the matrix. In addition, fiber surface modification by $\mathrm{NaOH}$ was employed for better bonding.

\section{Experimental}

\subsection{Materials}

Polypropylene (PP) supplied by Honam Petrochemical Corp. (Korea) was used as the matrix. The specific weight, the melting point, and the molecular weight of PP were $0.95 \mathrm{~g} / \mathrm{cm}^{3}, 170^{\circ} \mathrm{C}$, and $10,000 \mathrm{~g} / \mathrm{mol}$, respectively. The coupling agent was Maleic anhydride grafted polypropylene (MAPP) (Eastman, USA), which had an average molecular weight of 40,000. MAPP contains $6 \mathrm{wt} \%$ of maleic anhydride. Jute fibers, which were imported from the Philippines, were used as reinforcement. The lengths of the fibers were about 8-10mm. Analytical grade $\mathrm{NaOH}(98 \%)$ from Kudko. Co. (Korea) was used in the treatment of the jute fibers.

\subsection{Jute fiber surface treatments}

$\mathrm{NaOH}$ treatment was used in the delignification of the jute fibers. The treatment involved the use of a $2 \mathrm{wt} \%$ solution of $\mathrm{NaOH}$, where the fiber:solution ratio was 1:6 (by weight). Then, the jute fibers and the appropriate pre-mixed alkali solutions were mixed in a $5 \mathrm{~L}$ glass beaker and it was kept for 24hours. After treatment, the fibers were washed with rinsing tap water and finally thoroughly washed with running distilled water several times, and after every wash the $\mathrm{pH}$ level was measured. $\mathrm{pH}$ level 7 was maintained in the fiber solution. The fibers were then vacuum dried at $70^{\circ} \mathrm{C}$ for 24 hours.

\subsection{Composite fabrication}

Treated jute fibers were chopped into short lengths of about $2 \mathrm{~mm}$ to ensure an easy blending. The chopped jute fibers and PP were than compounded with the coupling agent in a manual mixer. As the density of the fibers and matrix were known, the fiber volume fraction was calculated. The volume percentage of jute fiber in the blend was $10 \%$. In the case of MAPP contents, it varied from 1 to 3 $w t \%$ on the basis of PP content. The mixed blends were molded in a twin-screw extruder (PRIM TSC 16TC, Thermo Electron Corp.) to form pellets of diameter $1.0 \mathrm{~mm}$ by using the melt mixing process. The temperatures of five extruder sections from the first heating zone to the die were set as 180, 200, 210, 200, and $180^{\circ} \mathrm{C}$, respectively. The hopper rate, the screw and speed, and the speed of roll were adequately adjusted for this process. After extrusion, the pellets were vacuum dried at $70^{\circ} \mathrm{C}$ for 24 hours. The dried pellets were used to make the dogbone specimens for tensile testing in accordance to the ASTM standard using 
injection molding equipment. In addition, the specimens of pure PP were also prepared using the injection molding technique.

The tensile tests were carried out according to the ASTM D 638 Type I standard (dog-bone specimens) using a Universal Testing Machine (RB 301 Unitech M). The gage length was $25 \mathrm{~mm}$ and the crosshead speed was $2.50 \mathrm{~mm} / \mathrm{min}$. At the same time, strain was measured by an extensometer over a gage length of $25.0 \mathrm{~mm}$. The tensile modulus, tensile strength, and percentage of elongation at break were computed from the stress-strain curves.

\section{Results and discussion}

The typical tensile strength-strain curves for tensile test specimens of the composites are shown in Figure 1. It shows the effect of jute fibers and coupling agent on the tensile strengths of pure and reinforced PP. In addition, the results of the tensile test are summarized in Table 1. The improvement of tensile strengths of the composites can be observed from Figure 1. The addition of 1 wt $\%$ MAPP increased the tensile strength of the composites from 20.28MPa to 24.19MPa. The improvement of mechanical properties by adding MAPP was occurred through a better bonding between cellulosic fiber surfaces and PP, was caused by the esterification of the anhydride groups of MAPP with the hydroxyl groups of cellulosic fibers. Because of the fact that jute fiber is lignocellulosic and contains more than $60 \%$ cellulose, we assume that a similar chemical bonding occurred between the hydroxyl groups of jute fibers and anhydride

Table 1: $\quad$ Tensile properties of jute/PP composites.

\begin{tabular}{c|c|c|c}
\hline Specimen & $\begin{array}{c}\text { Young's } \\
\text { modulus (GPa) }\end{array}$ & $\begin{array}{c}\text { Tensile strength } \\
(\mathrm{MPa})\end{array}$ & $\begin{array}{c}\text { Elongation at } \\
\text { break (\%) }\end{array}$ \\
\hline Pure PP & 0.75 & 20.28 & 19.38 \\
\hline Jute/PP (1\%MA) & 1.58 & 24.19 & 8.19 \\
\hline Jute/PP (2\%MA) & 1.67 & 24.68 & 8.29 \\
\hline Jute/PP (3\%MA) & 1.86 & 25.11 & 8.57 \\
\hline
\end{tabular}

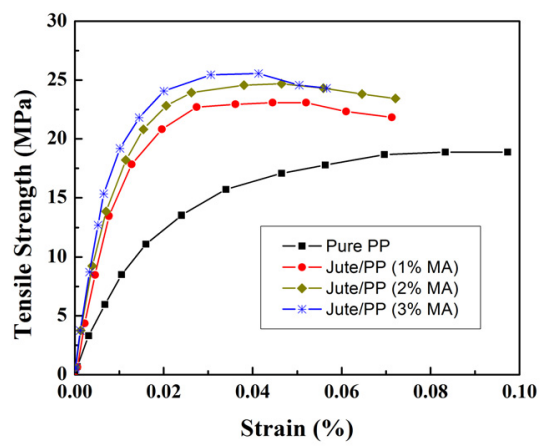

Figure 1: Tensile strength of jute/PP composites. 
groups of MAPP. Moreover, it was observed that the tensile strengths of the composites increased with the addition of MAPP content from $1 \%$ up to $3 \%$. The tensile strengths of the composites containing $2 \mathrm{wt} \%$ and $3 \mathrm{wt} \%$ MAPP contents were found to be $24.68 \mathrm{MPa}$ and $25.11 \mathrm{MPa}$, respectively.

Figure 2 shows the tensile modulus of jute/PP composites. It was observed that the elastic moduli were remarkably higher than those of pure PP, and they were almost independent of whether coupling agent was added or not. The moduli were determined from the initial slope of the stress-strain curve. At the initial slope, only a very small strain region can be considered, which is practically not influenced by the interface between fiber and matrix.

Variation of elongation at break with jute fiber composites containing different MAPP contents shows elongation of break of PP significantly reduced by the inclusion of jute fibers as shown in Figure 3. The decreases can be explained by constrained matrix flow in the presence of less-extensible fibers and the effects of internal stress concentrations initiated by fiber-induced microcracks [24]. However, in contrast to the normal elongation behavior of most synthetic [25, 26] and natural [27] plant based fiber-reinforced composites, in which elongation of break decreases continually with increasing fiber content. However, the percentage elongations of break of the jute fibers composites were increased with the increasing of MAPP content from $1 \%$ to $3 \%$.

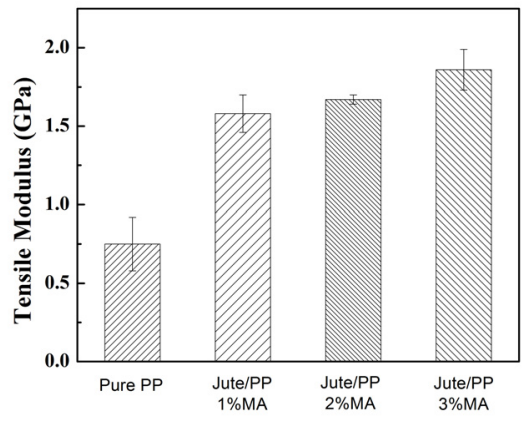

Figure 2: Tensile modulus of jute/PP composites.

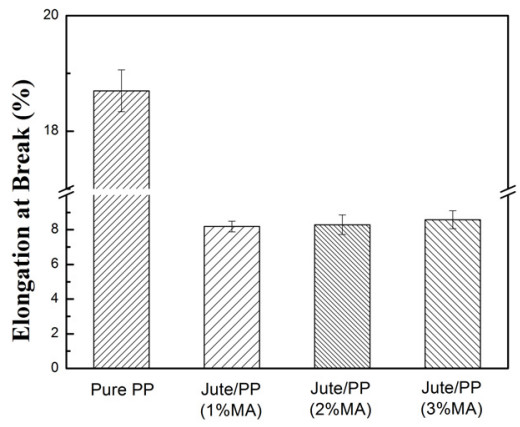

Figure 3: Elongation at break of jute/PP composites. 


\section{Conclusions}

Jute fiber reinforced polypropylene matrix composites were successfully developed by the injection molding technique. The improvement of tensile strengths and modulus of the composites can be observed. The addition of MAPP contents as coupling agent improved the composite performance by enhancing the adhesion between jute fibers and PP although the fiber volume percentage was only $10 \%$. As the elongations at breaks were reduced in jute fiber polypropylene composites, it can be suitable candidates for strain improvement in hybrid composite systems.

\section{Acknowledgements}

The authors would like to gratefully acknowledge the support from the Korea Foundation for International Cooperation of Science \& Technology (KICOS) through a grant provided by the Korean Ministry of Education, Science \& Technology (MEST) in 2007 (No. K20704000090). The authors would also like to acknowledge the partial support from the Second stage of Brain Korea 21 Project Corps for carrying out this work.

\section{References}

[1] Khondker, O. A., Ishiaku, U. S., Nakai, A., and Hamada, H., A novel processing technique for thermoplastic manufacturing of unidirectional composites reinforced with jute yarns, Composites: Part A, 37, pp. 22742284, 2006.

[2] Abdelmouleh, M., Boufi, S., Belgacem, M. N., and Dufresne, A., Short natural-fibre reinforced polyethylene and natural rubber composites: Effect of silane coupling agents and fibres loading, Composites Science and Technology, 67, pp. 1627-1639, 2007.

[3] Wulin, Q., Takashi, E., and Takahiro, H., Structure and properties of composites of highly crystalline cellulose with polypropylene: Effects of polypropylene molecular weight, European Polymer Journal, 42, pp. 10591068, 2006.

[4] Andrzej, K. B., and Faruk, O., Injection moulded microcellular wood fibrepolypropylene composites, Composites: Part A, 37, pp. 1358-1367, 2006.

[5] Mariano, P., Donatella, C., Irene, A., Zbigniew, K., and Poirkowska, E., Functionalization compatibilization and properties of polypropylene composites with Hemp fibres, Composites Science and Technology, 66, pp. 2218-2230, 2006.

[6] Gatenholm, P., and Felix, J., Wood fiber/polymer composites: fundamental concepts, process, and material options. Madison: Forest Product Society, 1993. 
[7] Carvalho, L. H., Leao, A. L., Carvalho, F. X., and Frollini, E., Lignocellulosic-plastics composites. Brazil: USP and UNESP, 1997.

[8] Belgacem, M. N., and Gandini, A., The surface modification of cellulose fibers for use as reinforcing elements in composite materials, Comp Inter, 24(1-2), pp. 41-75, 2005.

[9] Park, J. M., Son, T. Q., Byung, S. H., and Lawrence, K. D., Interfacial evaluation of modified Jute and Hemp fibers/polypropylene (PP)-maleic anhydride polypropylene copolymers (PP-MAPP) composites using micromechanical technique and nondestructive acoustic emission, Compos Sci Tech, 66, pp. 2689-2699, 2006.

[10] Panthapulakkal, S., Sain, M., and Law, L., Effect of coupling agents on rice husk-filled HDPE extruded profiles, Polym Int, 54, pp. 137-142, 2005.

[11] Keener, T. J., Stuart, R. K., and Brown, T. K., Maleated coupling agents for natural fiber composites. Composites Part A, 35, pp. 357-362, 2004.

[12] Botaro, V. R., and Gandini, A., Chemical modification of the surface of cellulosic fibers. 2. Introduction of alkenyl moieties via condensation reactions involving isocyanate functions, Cellulose, 5(14), pp. 65-78, 1998.

[13] Wulin, Q., Farao, Z., Endo, T., and Hirotsu, T., Isocyanate as a compatibilizing agent on the properties of highly crystalline cellulose/polypropylene composites, J Mater Sci, 40, pp. 3607-3614, 2005.

[14] George, J., Sreekala, M. S., and Thomas, S., A review on interface modification and characterization of natural fiber reinforced plastic composites, Polym Eng Sci, 41(9), pp. 1471-1485, 2001.

[15] Zadoreki, P., and Flodin, P., Surface modification of cellulose fibers. I. Spectroscopic characterization of surface-modified cellulose fibers and their copolymerization with styrene, J Appl Polym Sci, 30, pp. 2419-2429, 2003.

[16] Colom, X., Carrasco, F., Pagesc ,P., and Canavate, J., Effects of different treatments on the interface of HDPE/lignocellulosic fiber composites, Compos Sci Tech, 63, pp. 161-169, 2003.

[17] Mohd Ishak, Z. A., Aminullah, A., Ismail, H., and Rozman, H.D., Effect of silane-based coupling agents and acrylic acid based compatibilizers on mechanical properties of oil palm empty fruit bunch filled high density polyethylene composites, J Appl Polym Sci, 68, pp. 2189-2203,1998.

[18] Demir, H., Atiklera, U., Balkosea, D., and Tihmınlıoglua, F., The effect of fiber surface treatments on the tensile and water sorption properties of polypropylene-luffa fiber composites, Composites A, 37, pp. 447-456, 2006.

[19] Jingshen, W., Demei, Y., Chi-ming, C., Jangkyo, K., Yiu-wing, M., Effect of fiber pretreatment condition on the interfacial strength and mechanical properties of wood fiber/pp composites, J Appl Polym Sci, 76, pp. 10001010, 2000.

[20] Valadez, G. A., Cervantes, U., Olayo, R., and Herrera-Franco, P., Chemical modification of henequen fibers with an organosilane coupling agent, Composites B, 30, pp. 321-331, 1999. 
[21] Felix, J. M., and Gatenholm, P., The nature of adhesion in composites of modified cellulose fibers and polypropylene, Journal of Applied Polymer Science, 42, pp. 609-620, 1991.

[22] Karmaker, A. C., and Youngquist, J. A., Injection Molding of Polypropylene Reinforced with Short Jute Fibers, Journal of Applied Polymer Science, 62, pp. 1147-1151, 1996.

[23] Chen, X., Guo, Q., and Mi, Y., Bamboo Fiber-Reinforced Polypropylene Composites: A Study of the Mechanical Properties, Journal of Applied Polymer Science, 69, pp. 1891-1899, 1998.

[24] Bader, M. G., and Hill, A. R., Structure and properties of composites-short fiber composites, New York: VCH Publishers Inc., 1991.

[25] Lu, Y., Mechanical properties of random discontinuous fiber composites manufactured from wetlay process, engineering science and mechanics, Blacksburg: Virginia Polytechnic Institute and State University, pp. 116, 2002.

[26] Caba, A. C., Characterization of carbon mat thermoplastic composites: flow and mechanical properties, engineering science and mechanics, Blacksburg: Virginia Polytechnic Institute and State University, pp. 145, 2005.

[27] Angles, M. N., Salvado, J., and Dufresne, A., Steam-exploded residual softwood-filled polypropylene composites, J Appl Polym Sci, 74(8), pp. 1962-1977, 1999. 